\title{
Is promotion of blood donation in the GUM clinic all in vein?
}

Your Hospital

University Hospitals of South Manchester Foundation NHS Trust, Department of Sexual Medicine \& HIV, Manchester, United Kingdom

Background: In the UK less than $3 \%$ of the eligible* population donate blood ${ }^{1}$. The blood donation service faces a constant challenge of signing up new donors; with a reported $40 \%$ fewer new volunteers in 2014/15 compared with a decade ago $^{2}$ and stocks remain at critically low levels. There is a marked challenge in recruiting people from Black and minority ethnic (BME) communities, who account for around $14 \%$ of the UK population ${ }^{3}$, but only make up $5 \%$ of donors ${ }^{1}$. BME are more likely to have blood transfusion dependent conditions, such as thalassaemia and sickle cell disease, therefore recruiting in this group is particularly important. Genitourinary Medicine (GUM) is unique in secondary care; in having a predominantly young and healthy population. The histories we take in GUM clinics match closely with screening questions asked by the donation service and we wanted to explore whether there would be any value in utilising this similarity in promoting blood donation to our patients.

Methods: We conducted a prospective review of 100 consecutive patients seen during clinic, adding one extra question (regarding recent travel) to our usual history proforma to match the screening questions.

Results:

\section{Demographics}

Male:Female: 49:51 Age Range: 17 - 70 Median Age: 32 Eligibility for donation
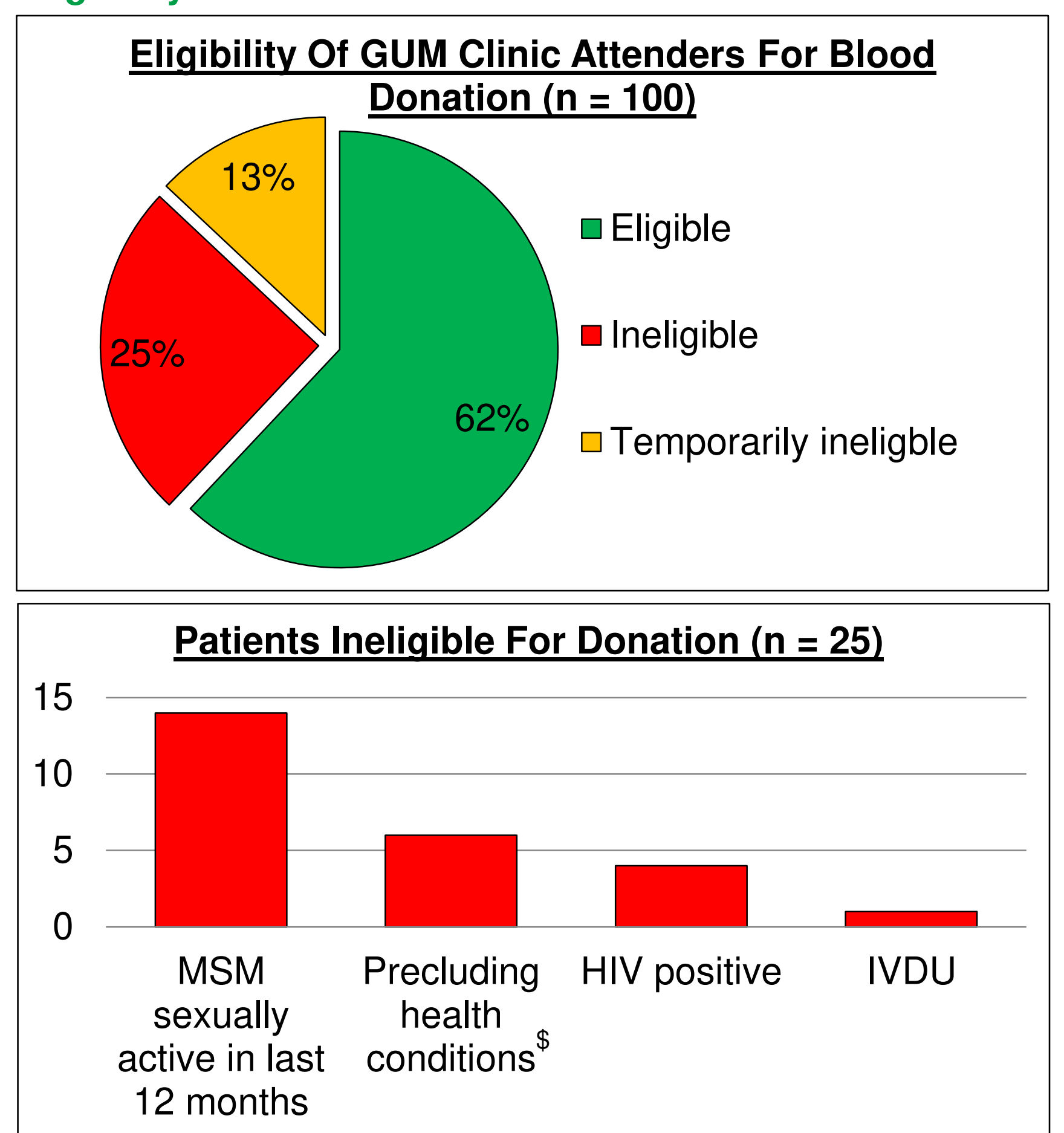
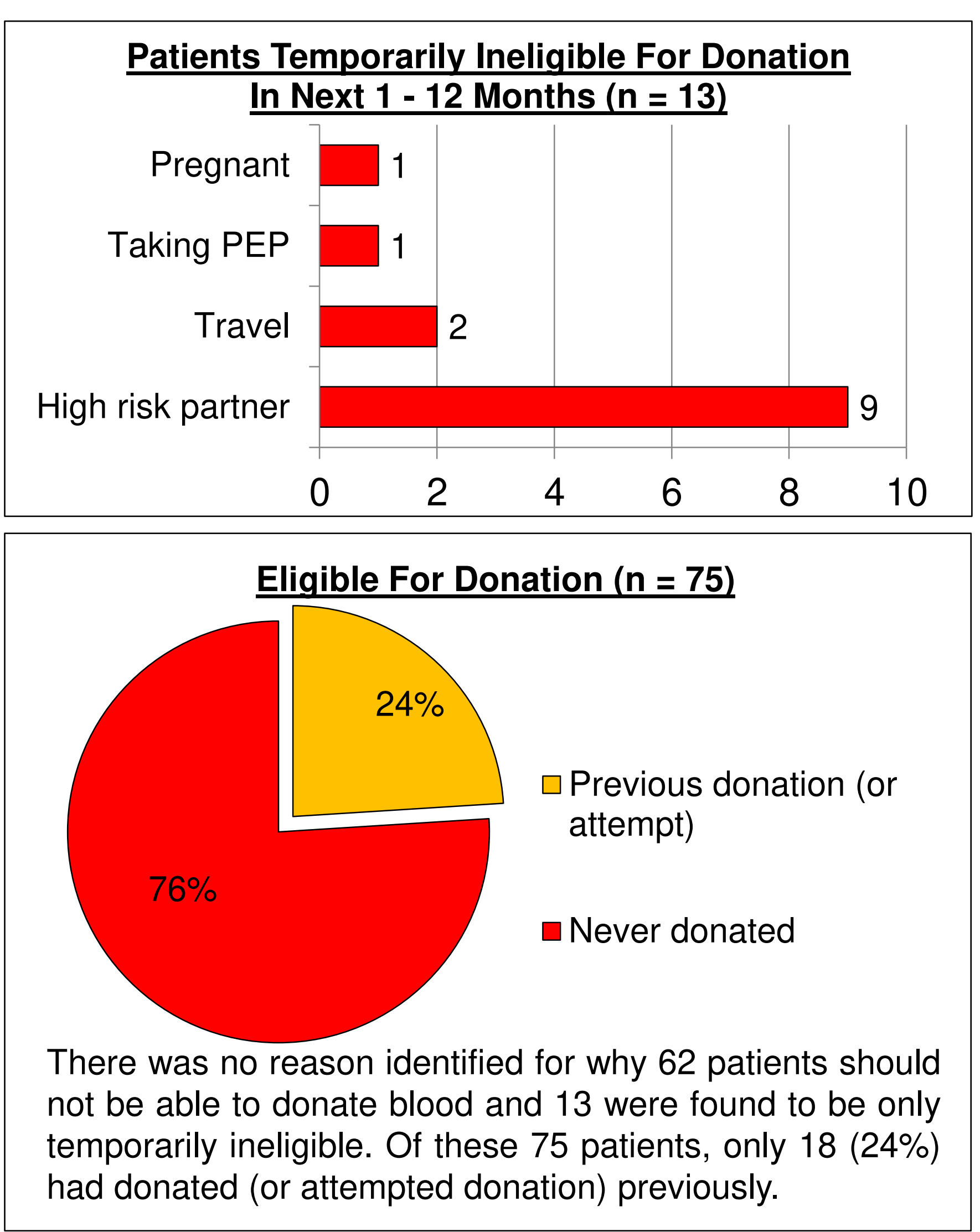

\$Precluding health conditions: four patients previously treated for cancer (ovarian, breast, renal, cervical), one patient with type 2 diabetes requring insulin, one patient diagnosed with rheumatoid arthritis taking hydroxychloroquine.

Discussion: GUM clinics may erroneously be perceived to be a counterintuitive location to recruit new donors, due to 'high risk' patients, however we found that $75 \%$ of the patients seen were potentially eligible. There have been reports that a working group for the Advisory Committee on the Safety of Blood, Tissues and Organs (SaBTO) will likely pass on recommendations to the Department of Health later this year, to reduce the deferral periods from 12 to 3 months for sexually active MSM, which would see our eligibility figure increase further. Our clinic has a BME population of $25 \%$, higher than the proportion nationally, thus providing an opportunity to promote donation to this under-represented group.

No additional time was needed to identify potential donors and only a brief intervention or posters in the clinic could be used to promote or signpost blood donation. We recognise that these figures give only an indication as to whether a patient would be eligible for blood donation; our questions were only based on initial screening questions, and we did not take into consideration factors such as patients' weight or occupation, for example. This was also a small pilot. We intend to liaise with NHS Blood and Transplant regarding promotional strategies and plan to repeat the study with a larger sample.

References: 1. blood.co.uk [Accessed 23/05/2017] 2. NHS Blood and Transplant Survey 2015 3. Office for National Statistics, 2011 Census *Eligibility only based on age (17-70), does not factor in medical history, or lifestyle factors etc. 